\title{
Design of An Intelligent Car Based on MSP430
}

\author{
Ruming Kang, Fengzhi Dai * \\ Tianjin University of Science and Technology, Tianjin, China \\ E-mail: *daifz@tust.edu.cn \\ www.tust.edu.cn
}

\begin{abstract}
The system takes the MSP430 single chip microcomputer as the control core, uses the reflective photoelectric sensor TCRT5000 module to track the line, and realizes the automatic tracking. In the experiment, the black-and-white line is used as the route. The system is driven by the L298N module and controlled by the PWM DC motor. This paper introduces the principle of the reflective photoelectric sensor and the circuit diagram of the tracking module. It also explains how to realize the automatic tracking based on the MSP430 single chip microcomputer. The technology can be used in warehouse, unmanned production line, intelligent service robot and other fields.
\end{abstract}

Keywords: MSP430, Sensor, Tracking module, Motor driven

\section{Introduction}

This smart car material comes from the 2020 National University Student Electronic Design Competition. It used TI's MSP430 microcontroller as the control center of the four-wheeled car, and transmits the signals of each sensor to the microcontroller for analysis and processing, thereby controlling the L $298 \mathrm{~N}$ motor drive, controlling the car and buzzer the device tweets.

The car is required to be able to follow the designated route and automatically follow the line on the ramp. The car must run independently, and no equipment (including power supply) can be used outside the car. The weight of the trolley (including battery) is less than $1.5 \mathrm{~kg}$, and the outline dimension on the ground is not more than $25 \mathrm{~cm} \times$ $25 \mathrm{~cm}$. The ramp is made of blockboard with a length and width of about $1 \mathrm{~m}$, allowing the natural color and natural wood grain on the board.

The surface of the wood board is laid with $1 \mathrm{~cm} \times 1 \mathrm{~cm}$ black and white paper strips (hereinafter referred to as the marking line) as route instructions; the starting section of the marking line is a straight line, parallel to both sides of the board; the marking line turns $90^{\circ}$ at the top of the slope, and the turning radius is $20 \mathrm{~cm}$; The distance between the marking line parallel to the top of the slope is $\geqslant 30 \mathrm{~cm}$, and the distance from the top of the slope is $\leqslant 20 \mathrm{~cm}$; the total length of the marking line is $1 \mathrm{~m}$. The parking mark is a black line with a width of $1 \mathrm{~cm}$ and a length of $5 \mathrm{~cm}$, perpendicular to the marking line on the top of the slope. The slope angle of the trolley and the top view of the driving route are shown in Fig.1.

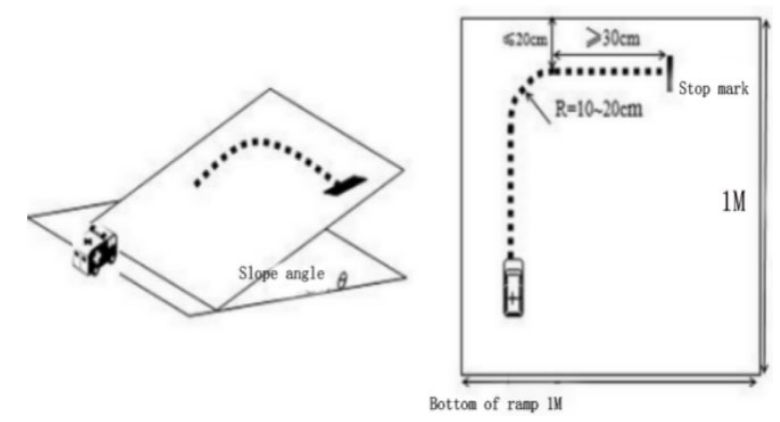

Fig.1 Schematic diagram of car ramp angle and top view of driving route

(C) The 2021 International Conference on Artificial Life and Robotics (ICAROB2021), January 21 to 24, 2021 


\section{Modularization of the car hardware}

The smart car can be divided into: power module, sensor detection module, microcontroller module and DC motor drive module. The smart car can realize functions such as tracking and parking. It uses the MSP430 single-chip microcomputer as the main control chip, and uses a DC motor as the power. The hardware system structure diagram is shown as in Fig.2.

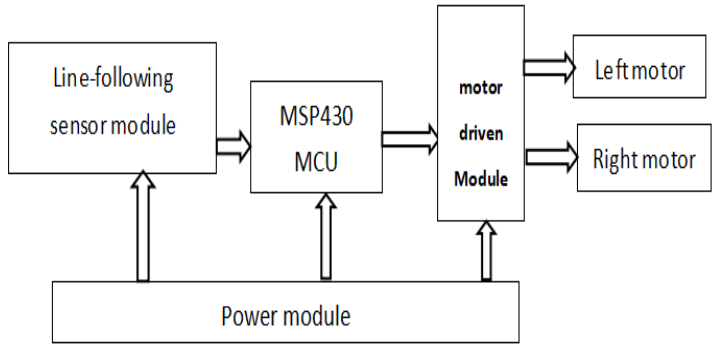

Fig.2 Hardware system structure diagram

\subsection{Motor drive module}

The motor adopts a PWM DC motor, which can be used as an ordinary motor (no coding function is used to make it have better mechanical speed regulation performance, and the DC-DC step-down voltage stabilized power supply module is used to realize the mechanical speed regulation of the motor), and it is driven by L298N.

The single-chip microcomputer controls the level of the L298N enable terminal to realize the rotation of the motor and realize the forward or steering of the trolley. The principle of the direction change of the DC motor is: if turning left, the left wheel will not move, and the right wheel will turn; if turning right, the opposite; if going straight, both left and right wheels will turn. But the problem that 298 chips are prone to heat is difficult to solve. The physical picture of L298N is shown in Fig.3.

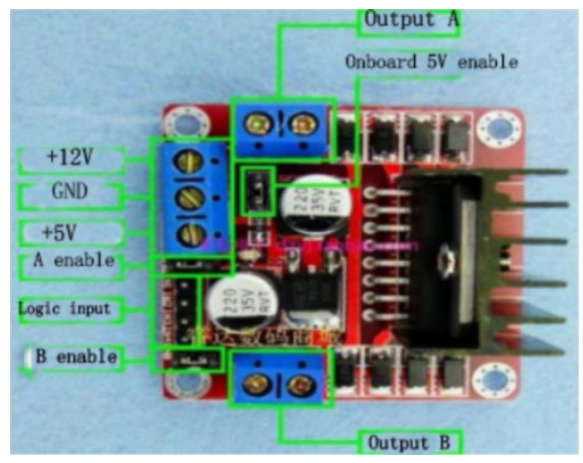

Fig.3 Physical Picture of L298N Module

\subsection{Tracking module}

Component: CRT5000 photoelectric sensor

Principle: The infrared emitting diode of the TCRT5000 sensor continuously emits infrared rays. When the emitted infrared rays are not reflected back or reflected back but the intensity is not strong enough, the infrared receiving tube is always in the off state, and the output terminal of the module is high at this time. Indicating that the diode is always off; when the detected object appears in the detection range, the infrared is reflected back and the intensity is large enough, the infrared receiving tube is saturated, and the output of the module is low at this time, indicating that the diode is lit.

The application in the actual program is: two TCRT5000 sensors are installed on the left and right sides of the car. (1) When the left and right sensors are in the white area, that is, the emitted infrared rays are emitted back, both the left and right motors rotate. (2) When only the left sensor detects the black line, the infrared is not reflected back, indicating that the car is driving to the right. At this time, the motor on the right rotates and the motor on the left stops, and the car shows a left turn correction. (3) When the right sensor detects a black line, the emitted infrared is not reflected back, indicating that the car is to the left. The motor on the left rotates and the motor on the right stops, and the car turns right to correct.

The schematic diagram of the tracking process and the circuit diagram of the tracking sensor module are shown in Fig.4 and Fig.5.

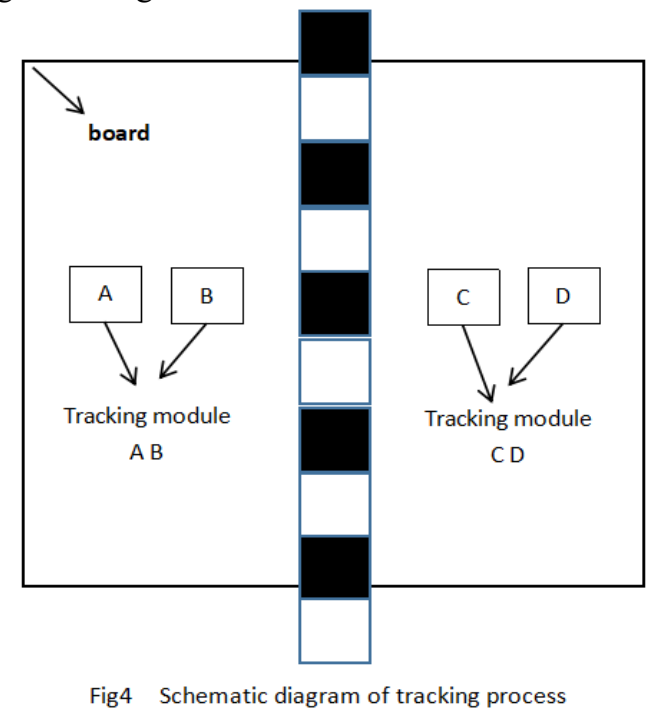

(C) The 2021 International Conference on Artificial Life and Robotics (ICAROB2021), January 21 to 24, 2021 


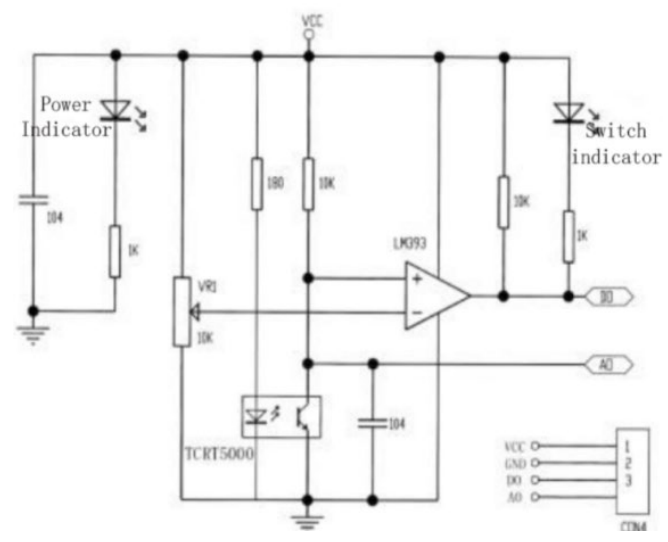

Fig.5 Schematic diagram of car ramp angle and top view of driving route

\subsection{Power Module}

The power module is divided into two parts: one is the chip power module, which uses a $4.2 \mathrm{~V}$ lithium battery to drive the chip; the other is the motor drive module, which provides higher power to drive the motor through L298N.

Motor drive power supply: Use two $4.2 \mathrm{~V}$ lithium batteries connected in series to form about $8.4 \mathrm{~V}$ to provide power to the L298N chip. Generally, the battery has high power, is more stable and durable, can be charged more economically, and has better stability, and is more convenient to use.

Chip drive power supply: Since the power requirement is not large, but the stability is high, so use the 8.4 voltage regulator circuit to drive (the voltage drop has been considered, of course, the voltage drop of each electronic device is different, and you need to debug according to your own.

\section{System software design}

The smart car is based on the modular design concept, and the software design corresponds to the hardware system, mainly for tracking subroutine modules and parking subroutine modules. The main program calls these subroutine modules in logical order.

The single-chip microcomputer collects the signals collected by the sensors of each module, after processing, and then controls the actions of the trolley through the motor driver to complete the predetermined target. When the single-side sensor detects the black line, it judges the status according to the tracking subroutine and makes steering adjustment. When both the line-following sensors detect the black line, it judges the turning state according to the parking subroutine and makes a parking action.

If it reflects that the obstacle avoidance and parking actions are inaccurate or there are loopholes, you can appropriately add line-following sensors on both sides, and make corresponding modifications in the subroutine to improve the accuracy of obstacle avoidance and parking. The obstacle avoidance flowchart is shown in the Fig. 6.

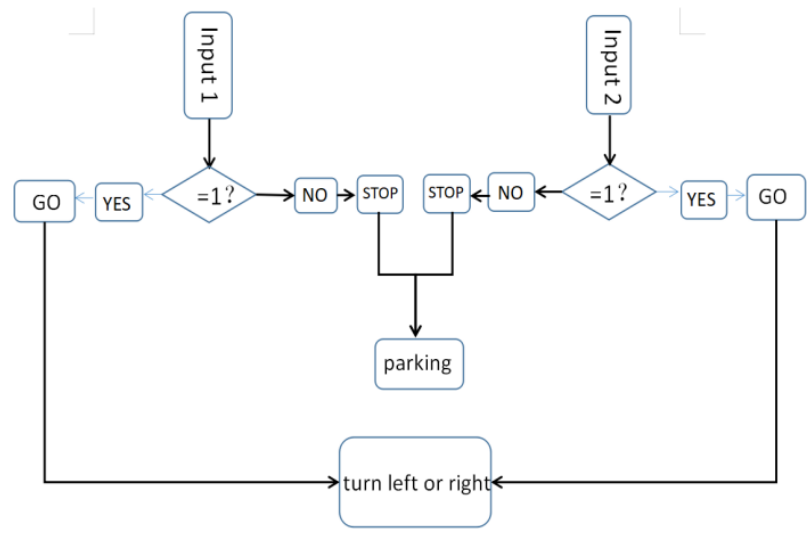

Fig.6 System software design flow chart

\section{Conclusion}

This article explains the intelligent car control system based on MSP430 single chip microcomputer chip. Through the infrared tracking sensor, the car tracking and parking functions are realized, and the expected design effect is achieved. In the design process, light has an occasional impact on the tracking. LED lights can be installed adjacent to the line-following sensor to enhance the light and strengthen the recognition of black lines. At the same time, the tracking algorithm should be optimized to make the tracking more accurate.

\section{References}

1. Ying Ceng, He Ming Zhao, Zhi Ling Peng, Design of Multifunctional Intelligent Car Based on MSP430, Electronic Measurement Technology, 2017 (6): pp. 10-14.

2. Meng ting Chen, Bai yan $\mathrm{Hu}$, Can Huang, Design of Intelligent Tracking and Obstacle Avoidance Car Based on Single Chip Computer, Smart Robot, 2016(4): pp.47.

3. Zheng Bing Wan, Design of automatic tracking car based on LDC1000, Automation technology, 2016(10): pp. 111-157.

4. Qiu Yan Zhang, Jun Xia Zhang,Yang Yang, Design of Multifunctional Intelligent Car Based on MSP430, Information Technology, 2018(11): pp.54-58. 